TITLE:

\title{
Dynamical aspects of an adiabatic piston
}

AUTHOR(S):

Munakata, T; Ogawa, $\mathrm{H}$

CITATION:

Munakata, T ... [et al]. Dynamical aspects of an adiabatic piston. Physical Review E 2001, 64(3): 036119.

ISSUE DATE:

2001-09

URL:

http://hdl.handle.net/2433/50308

RIGHT:

Copyright 2001 American Physical Society 
PHYSICAL REVIEW E, VOLUME 64, 036119

\title{
Dynamical aspects of an adiabatic piston
}

\author{
Toyonori Munakata and Hideki Ogawa \\ Department of Applied Mathematics and Physics, Graduate School of Informatics, Kyoto University, Kyoto 606-8501, Japan
}

(Received 23 February 2001; published 29 August 2001)

\begin{abstract}
Dynamical aspects of an adiabatic piston are investigated, based on the mass ratio expansion of the master equation for the piston velocity distribution function. Simple theory for piston motion and relaxation of an ideal gas in a cylinder turns out to reproduce our numerical experiments quantitatively.
\end{abstract}

DOI: 10.1103/PhysRevE.64.036119

The adiabatic piston has gathered considerable attention recently, partly in connection with thermodynamics and kinetics $[1,2]$ to predict transient (relaxational) processes and the final equilibrium state and partly its apparent relation to the ratchet dynamics [3]. In short the problem is how an adiabatic piston, which separates a gas-filled cylinder into two parts with different pressure and/or temperature, moves and how the whole system relaxes towards its equilibrium state. Since we are interested in dynamics of the whole piston-gas system, we study the problem based mainly on the master equation [4] for the piston velocity distribution function $p(V, t)$ and also on thermodynamic considerations to deal with relaxation processes in the piston-gas system.

Our system consists of a cylinder with length $L$ and a piston with mass $M$, which separates the cylinder into the left and right parts $(i=l, r)$, each containing $N_{i}(i=l, r)$ ideal gas particles with mass $m$. The density $n_{i}(i=l, r)$ is given by

$$
n_{l}=N_{l} /(X A), \quad n_{r}=N_{r} /[(L-X) A],
$$

where $X$ is the length of the left cell and $A$ denotes the cross sectional area of the cylinder. To derive a master equation for the piston velocity distribution function $p(V, t)$, let us consider the collision between the piston with velocity $V$ and a particle with velocity $v$ in the left cell. From the conservation of energy and momentum, the velocity after the collision $V^{\prime}$ of the piston is

$$
V^{\prime}=V+2 m(v-V) \Theta(v-V) /(M+m),
$$

where the step function $[\Theta(x)=1(0)$ for $x>0(x<0)]$ shows that the collision is possible only for the case $v>V$. Assuming that the velocity distribution of the ideal gas in the left cell is Maxwellian with temperature $T_{l}$, the transition rate $W_{l}\left(V \rightarrow V^{\prime}\right)$ of the piston velocity from $V$ to $V^{\prime}$ is given by

$$
\begin{aligned}
W_{l}\left(V \rightarrow V^{\prime}\right)= & n_{l}[(M+m) /(2 m)]^{2}\left(V^{\prime}-V\right) A f_{M} \\
& \times\left(\left[\{M+m\} V^{\prime}-\{M-m\} V\right] /\{2 m\} ; T_{l}\right) \\
& \times \Theta\left(V^{\prime}-V\right)
\end{aligned}
$$

with

$$
f_{M}(v ; T)=(2 \pi T / m)^{-1 / 2} \exp \left[-m v^{2} /(2 T)\right] .
$$

PACS number(s): 02.50.-r, 05.40.-a, 05.20.-y

Here and hereafter we set the Boltzmann constant equal to 1 . Similarly the transition rate due to a collision with a particle in the cell $r$ is given by

$$
\begin{aligned}
W_{r}\left(V \rightarrow V^{\prime}\right)= & n_{r}\left[(M+m)^{2} /(2 m)^{2}\right]\left(V-V^{\prime}\right) A f_{M} \\
& \times\left(\left[\{M+m\} V^{\prime}-\{M-m\} V\right] /\{2 m\} ; T_{r}\right) \\
& \times \Theta\left(V-V^{\prime}\right) .
\end{aligned}
$$

Since the piston velocity $V$ changes due to collisions with particles in both the right and left cells, the (total) transition rate $W\left(V \rightarrow V^{\prime}\right)$ is defined by $W\left(V \rightarrow V^{\prime}\right) \equiv W_{l}\left(V \rightarrow V^{\prime}\right)$ $+W_{r}\left(V \rightarrow V^{\prime}\right)$, and we obtain the following master equation for the probability density $p(V, t)$ of the piston velocity:

$$
\begin{aligned}
\partial p(V, t) / \partial t= & \int d V^{\prime}\left[W\left(V^{\prime} \rightarrow V\right) p\left(V^{\prime}, t\right)\right. \\
& \left.-W\left(V \rightarrow V^{\prime}\right) p(V, t)\right] .
\end{aligned}
$$

Now we apply the mass ratio expansion $[4,5]$ method developed by van Kampen to transform the complicated integrodifferential equation (6) to a tractable Fokker-Planck equation. Referring the details of the derivation to the original paper [5], we only give an essential idea behind the mass ratio expansion. Introducing the smallness parameter $\epsilon$ by

$$
\epsilon \equiv m /(m+M),
$$

we express velocity of the piston $V$ as the sum of its deterministic part $V_{D}$ and fluctuation $\epsilon^{1 / 2} u$ as

$$
V=V_{D}+\epsilon^{1 / 2} u \text {. }
$$

Next from Eqs. (2), (3), and (5), we notice that the transition rate $W\left(V \rightarrow V^{\prime}\right)$ can be expressed as $\epsilon^{-1} \widetilde{W}\left(V ;\left[V^{\prime}-V\right] / \epsilon\right)$ with $\widetilde{W}(V, a) \equiv(A a / 4)\left[n_{l} f_{M}\left(\{V+a / 2\} ; T_{l}\right) \Theta(a)-n_{r} f_{M}(\{V\right.$ $\left.\left.+a / 2\} ; T_{r}\right) \Theta(-a)\right]$ and this allows immediately a formal Taylor expansion of the master equation (6) [5]. For $V_{D}$ we have the following evolution equation:

$$
d V_{D}(t) / d t=\epsilon \alpha_{1}\left(V_{D}: T_{l}, T_{r}, X\right),
$$

where the $n$th jump moment $\alpha_{n}(n=1,2, \ldots)$ is defined as

$$
\begin{aligned}
\alpha_{n}\left(V: T_{l}, T_{r}, X\right)= & \int d a a^{n+1} A\left[n_{l} f_{M}\left(V+a / 2: T_{l}\right) \Theta(a)\right. \\
& \left.-n_{r} f_{M}\left(V+a / 2: T_{r}\right) \Theta(-a)\right] / 4
\end{aligned}
$$


The $X$ dependence of $\alpha_{n}$ comes from the $X$ dependence of the density, see Eq. (1). For the fluctuation $u$ in Eq. (8) we have the Fokker-Planck equation

$$
\begin{aligned}
\partial p(u, t) / \partial t= & -\epsilon^{1 / 2} \partial / \partial u\left[\left\{\alpha_{1}\left(V_{D}+\epsilon^{1 / 2} u\right)-\alpha_{1}\left(V_{D}\right)\right\} p\right] \\
& +\sum_{n=2}\left\{\epsilon^{n / 2} /(n !)\right\}(-\partial / \partial u)^{n}\left[\alpha_{n}\left(V_{D}+\epsilon^{1 / 2} u\right) p\right] .
\end{aligned}
$$

Dynamics of $X(t)$ is naturally described by $d X / d t=V_{D}$ $+\epsilon^{1 / 2} u$. We employ a simplifying approximation in which the stochastic variable $X$ is replaced by its average over the distribution function $p(u, t)$, that is,

$$
d X / d t=V_{D}+\epsilon^{1 / 2}\langle u(t)\rangle .
$$

The method of mass ratio expansion has been mainly used for the case $T_{l}=T_{r}$ to study relaxation of the piston velocity to its Maxwellian equilibrium distribution [5]. We will use it to study the relaxation of the whole system (piston and gas) towards the equilibrium state.

From Eqs. (9) and (11) we see that the dynamics is determined by $\alpha_{n}$, Eq. (10). Although it is not difficult to calculate these coefficients numerically, we calculate some lowest order coefficients analytically for later convenience. Omitting $T$ and $X$ dependences we have

$$
\begin{aligned}
\alpha_{n}(V)= & B_{l} \int_{0}^{\infty} d a a^{n+1} \exp \left[m(a+2 V)^{2} /\left(8 T_{l}\right)\right] \\
& -B_{r} \int_{-\infty}^{0} d a a^{n+1} \exp \left[m(a+2 V)^{2} /\left(8 T_{r}\right)\right],
\end{aligned}
$$

where

$$
B_{i}=A n_{i}\left(m / 2 \pi T_{i}\right)^{1 / 2} / 4 \quad(i=l, r) .
$$

From this we find, after some calculation, that

$$
\begin{gathered}
\alpha_{1}(V=0)=A\left(n_{l} T_{l}-n_{r} T_{r}\right) / m=A\left(p_{l}-p_{r}\right) / m, \\
\begin{aligned}
\alpha_{2}(V=0) & =8 A(2 \pi)^{-1 / 2}\left[n_{l}\left(T_{l} / m\right)^{3 / 2}+n_{r}\left(T_{r} / m\right)^{3 / 2}\right] \\
& \equiv D_{0}(>0),
\end{aligned}
\end{gathered}
$$

where the equation of state for the ideal gas as used in Eq. (15) is

$$
p_{i}=n_{i} T_{i}(i=l, r) \text {. }
$$

As for the derivatives of $\alpha_{n}(V)$ with respect to $V$ similar calculations yield

$$
\begin{gathered}
\alpha_{1}^{\prime}(V=0)=-4 A\left[n_{l} T_{l}^{1 / 2}+n_{r} T_{r}^{1 / 2}\right] /(2 \pi m)^{1 / 2} \equiv-\zeta(<0), \\
\alpha_{2}^{\prime}(V=0)=6 A\left[-p_{1}+p_{2}\right] / m \\
\alpha_{1}^{\prime \prime}(V=0)=2 A\left[p_{l} / T_{l}-p_{r} / T_{r}\right] \equiv \sigma
\end{gathered}
$$

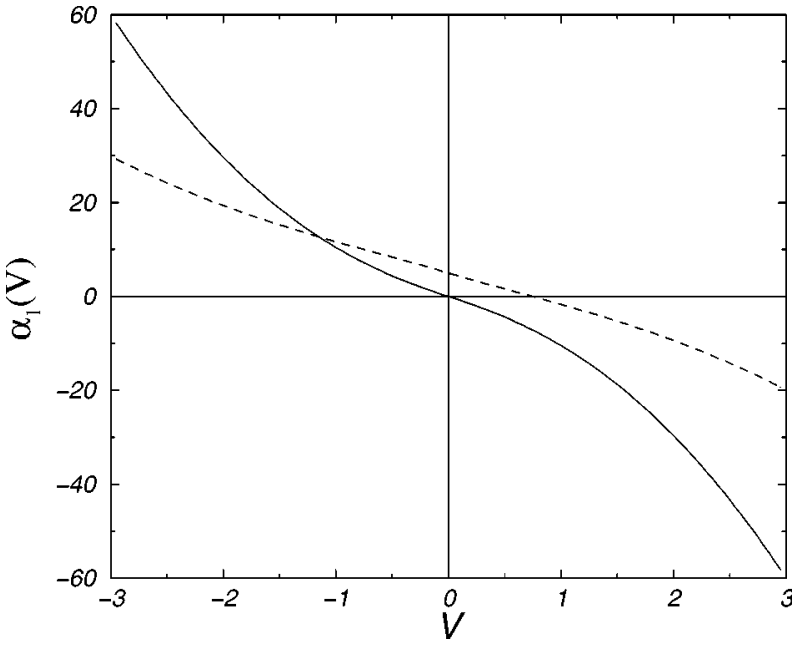

FIG. 1. Driving force $\alpha_{1}(V)$ for the cases $p_{l}>p_{r}$ (dotted curve, $T_{l}=3, n_{l}=2, T_{r}=1, n_{r}=1$ ) and $p_{l}=p_{r}$ (full curve, $T_{l}=3, n_{l}=1, T_{r}$ $\left.=1, n_{r}=3\right)$. The full curve goes through the point $(0,0)$ since $\alpha_{1}(0)=0$. It is noted that all the physical quantities to appear in this and subsequent figures are nondimensional, with units $m$ (the mass of an ideal gas perticle), the Boltzmann constant and $A$ (the cross sectional area of the cylinder).

$$
\begin{aligned}
\alpha_{1}^{\prime \prime \prime}(V=0) & =-4 A\left[n_{l} / \sqrt{T}_{l}+n_{r} / \sqrt{T}_{r}\right](m / 2 \pi)^{1 / 2} \\
& \equiv-\mu^{2}(<0)
\end{aligned}
$$

Furthermore we note that $\alpha_{2}^{\prime \prime}(V=0)$ $=12 \sqrt{2} A(m \pi)^{-1 / 2}\left[p_{1} / \sqrt{T}_{1}+p_{2} / \sqrt{T}_{2}\right]$ is a positive quantity. $\sigma$ defined by Eq. (20) may be considered to be symmetry breaking in the sense that even if the mechanical force balances, i.e., $p_{l}=p_{r}, \sigma$ does not necessarily vanish and can give rise to directional (toward the region of higher temperature) piston motion as shown below.

First we consider an infinite system $N_{l}, N_{r}=\infty$. In this case piston movement does not affect the thermodynamic conditions such as temperature and pressure and the problem is to obtain the stationary piston velocity. Hereafter in our numerical presentation we put $m=1$ and $A=1$ and all the quantities are nondimensional. In Fig. 1 we plot $\alpha_{1}(V)$ for the case $p_{l}=6\left(T_{l}=3, n_{l}=2\right)$, and $p_{r}=1\left(T_{r}=1, n_{r}=1\right)$ (dotted curve). The force on the piston due to the unbalance of the pressure $p_{l}$ and $p_{r}$ is represented by the ordinate of the point [see Eq. (15)] where the curve crosses the $y$ axis and the stationary velocity $V_{D}$ is given by the abscissa of the point where the curve crosses the $x$ axis in Fig. 1. The physical origin of $V_{D}$ becomes clear if we explicitly write down (to order $\epsilon^{3 / 2}$ ) the equation of motion (9) from Eqs. (15) and (18),

$$
(M+m) d V_{D} / d t=A\left(p_{l}-p_{r}\right)-m \zeta V_{D}
$$

Thus the stationary (or terminal) velocity $V_{D}$ is determined by the balance of the driving force and the frictional force. When $p_{l}=p_{r}$ the macroscopic velocity vanishes, $V_{D}=0$ (see Fig. 1 full curve) since $\alpha_{1}(V=0)=0$. In this situation, dynamics should be discussed based on Eq. (11). Taking the 


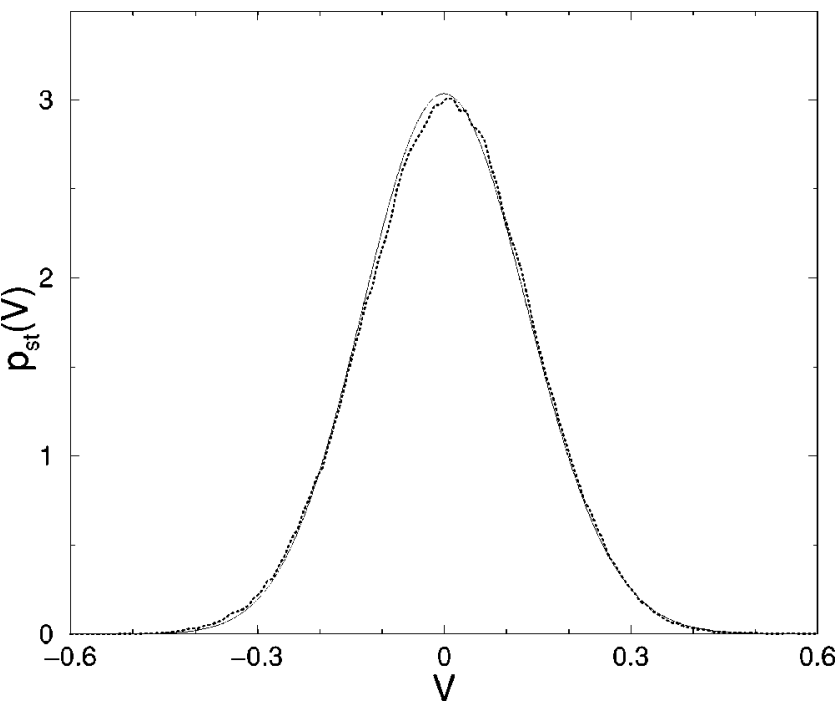

FIG. 2. Stationary distribution of the piston velocity for an infinite system from experiments (dotted curve) and theory (full curve).

dominant terms into consideration, we reduce Eq. (11) to the following Fokker-Planck equation

$$
\partial p(u, t) / \partial t=-\partial / \partial u[-\{d \Phi(u) / d u\} p]+\epsilon\left(D_{0} / 2\right)(\partial / \partial u)^{2} p,
$$

where we have defined the effective potential by

$$
\Phi(u)=\epsilon \zeta u^{2} / 2-(\sigma / 6) \epsilon^{3 / 2} u^{3}+\epsilon^{2} \mu^{2} u^{4} / 24 .
$$

Since thermodynamic parameters do not change in time for an infinite system we have a stationary distribution $p_{s t}(u)$ from Eq. (23)

$$
p_{s t}(u) \propto \exp \left[-\Phi(u) /\left(\epsilon D_{0} / 2\right)\right] .
$$

When $T_{l} \neq T_{r}$, the symmetry breaking parameter $\sigma$ does not vanish and the average $\langle u\rangle$ calculated by the distribution function (25) is not zero. More explicitly we can calculate perturbationally the average velocity to obtain [2]

$$
\sqrt{\epsilon}\langle u\rangle=\epsilon \sigma D_{0} /\left(4 \zeta^{2}\right)=\sqrt{(\pi / 8 m)}\left[\sqrt{T_{r}}-\sqrt{T_{l}}\right] \epsilon .
$$

Equation (26) shows that the piston moves in the direction of a hotter region in agreement with numerical experiments for a hard rod system [6] and an ideal gas as shown below.

Now we turn to a system with finite size, where piston movement results in variation of various thermodynamic parameters and the problem is to determine the piston position $X(t)$ as a function of time. For this purpose we examine the time evolution of the system with the main concern put on the piston motion $d X(t) / d t$. Since the piston is heavy and moves slowly we can regard the two cells to be homogeneous, characterized by $T_{i}(t)(i=l, r)$ and $n_{i}(t)(i=l, r)$ and we have the equation of motion for the piston from Eq. (26)

$$
d X(t) / d t=\sqrt{(\pi / 8 m)}\left[\sqrt{T}_{r}-\sqrt{T}_{l}\right] \epsilon .
$$

Since the piston energy in the conservation law

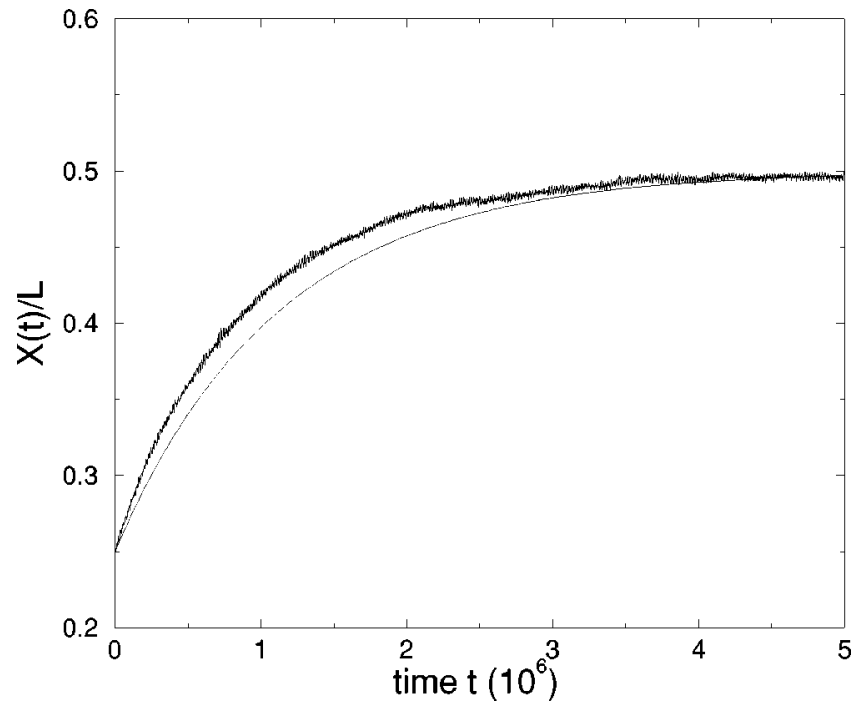

FIG. 3. Piston position $X(t)$ relaxing toward $X(t=\infty)=L / 2$. The curve with jags is the result of averages over 50 experiments and the full curve is from theory.

$$
\left(N_{l} / 2\right) d T_{l} / d t+\left(N_{r} / 2\right) T_{r} / d t+d\left[M\left\langle u^{2}\right\rangle / 2\right] / d t=0
$$

is negligible from Eq. (25) and under the condition $N_{i}(i$ $=l, r) \gg 1$, we have

$$
N_{l} d T_{l} / d t+N_{r} d T_{r} / d t=0
$$

Finally assuming that mechanical balance $p_{l}=p_{r}$ holds, we have

$$
N_{l} T_{l} / X=N_{r} T_{r} /(L-X) .
$$

From Eqs. (27), (28), and (29) it follows that

$$
d X / d t=-\sqrt{\left(\pi K_{0} / 8 m L\right)}[\sqrt{X}-\sqrt{(L-X) / c}] \epsilon,
$$

where $c \equiv N_{r} / N_{l}$ and $K_{0} \equiv T_{l}+c T_{r}$. Instead of showing the rather complicated analytic solution to Eq. (30), we consider the dynamics at the late stage when $X(t)$ approaches the equilibrium point $L /(1+c)$ and we easily obtain simple exponential behavior $\exp (-t / \tau)$ of $X(t)$ with the relaxation time $\tau$ given by

$$
\tau=(4 L c / \epsilon) \sqrt{2 m / \pi K_{0}(1+c)^{3}} .
$$

Simple exponential behavior, which was first observed in [6], is quantitatively verified below.

Finally in this theoretical part, we consider the thermodynamics of the system. First, from Eqs. (28) and (29) it is seen that the pressure Eq. (17) is a constant, which is in accord with our experimental observation (except for fluctuations). Since the entropy of a one-dimensional ideal gas is given by $S=N \ln [\sqrt{T} / n]$ to an additive constant, we have

$$
d\left(S_{l}+S_{r}\right) / d t=(3 / 2) N_{l}\left[T_{l}^{-1} d T_{l} / d t+c T_{r}^{-1} d T_{r} / d t\right],
$$

which is rewritten from Eq. (28) as 


$$
d\left(S_{l}+S_{r}\right) / d t=(3 / 2) N_{l}\left[T_{l}^{-1}-T_{r}^{-1}\right] d T_{l} / d t .
$$

From the second law we know that the right hand side is non-negative and heat is confirmed to flow from a high to a low temperature region. The same equation is derived if we apply the second law to, e.g., the left cell and study $d S_{l}$ $\geqslant d Q / T_{r}=\left(d E_{l}+p S d X\right) / T_{r}$.

Numerical experiments are rather simple for a system with finite size and a minor modification of a molecular dynamics method for a hard rod system [7] is enough, since we only need to consider elastic collisions of particles with a piston and a wall. In experiments for an infinite system, we take a Monte Carlo (MC) approach. First we choose a time increment $\Delta$ and calculate $z_{l} \equiv \Delta \int d V^{\prime} W_{l}\left(V \rightarrow V^{\prime}\right)$ and $z_{r}$ $\equiv \Delta \int d V^{\prime} W_{r}\left(V \rightarrow V^{\prime}\right)$ [see Eqs. (3) and (5)]. $z_{l}$, and $z_{r}$ denote the probabilities of the piston with velocity $V$ to collide with the left cell and right cell particles, respectively and 1 $-z_{l}-z_{r}$ is the probability of no collision. In order to avoid more than one collision in time $\Delta$ we choose $\Delta$ small enough so that $z_{l}+z_{r}<0.03$. If a collision with say, the left cell particle occurs, the new velocity $V^{\prime}$ is chosen according to the probability $W_{l}\left(V \rightarrow V^{\prime}\right) / \int d V^{\prime \prime} W_{l}\left(V \rightarrow V^{\prime \prime}\right)$. In Fig. 2 we plot the stationary velocity distribution $p_{s t}(V)$ (dotted curve), which is based on $8 \times 10^{6} \mathrm{MC}$ steps for an infinite system together with the theoretical one(full curve) $(\epsilon=0.01)$ for $T_{l}=1, n_{l}=0.1, T_{r}=3$, and $n_{r}=1 / 30$. The average velocity is 0.0059 from numerical experiments and 0.0046 from theory. It is noted that not only the mass ratio $\epsilon$ but the difference in the thermodynamic parameters of the two cells have effects on the validity of the theoretical prediction for $p_{s t}(V)$. Generally the smaller the $\epsilon$ and the differences in the thermodynamic parameters, the discrepancy in the stationary velocity distribution function between experiments and theory becomes small. For example, when $T_{l}$ and $T_{r}$ differ considerably, fluctuation in pressure becomes large and we have to take fluctuation effects into account. For the finite system we arbitrarily set $N_{l}=N_{r}=500(c=1)$, and $L=20000$. Experimental piston positions (jagged curve), which are averages over 50 experiments, and theoretical ones (smooth full curve) $X(t)$ are plotted in Fig. 3. The initial condition is $X(0) / L=0.25, T_{l}(0)=1$, and $T_{r}=3$. If we use Eq. (31) we have $\tau=1.1 \times 10^{6}$ and the best fit to experiments is achieved by a single exponential with $\tau=0.94 \times 10^{6}$.

Note added in proof. In the molecular dynamics simulation for a system with finite size, the velocity distribution of particles in an ideal gas is not necessarily Maxwellian and this seems to be one of the reasons for the discrepancies between our theory and experiments.

In this paper we studied the adiabatic piston problem based on the mass ratio expansion method. In spite of our neglection of some fluctuation and inhomogeneity effects, our theory turns out to reproduce the experimental results quantitatively. Equations (33) and (31) show that energy flows through the piston so long as the mass of the piston is finite. Thus from our treatment presented above we may say that the piston treated in this paper had better be called the Brownian piston.
[1] J. Piasecki and Ch. Gruber, Physica A 265, 463 (1999).

[2] Ch. Gruber and J. Piasecki, Physica A 268, 412 (1999).

[3] F. Juelicher, A. Ajdari, and J. Prost, Rev. Mod. Phys. 69, 1269 (1997); R. D. Astumian, Science 276, 917 (1997).

[4] C. W. Gardiner, Handbook of Stochastic Methods (Springer, Berlin, 1983).
[5] N. G. van Kampen, Can. J. Phys. 39, 551 (1961).

[6] E. Kestemont, C. van den Broeck, and M. M. Mansour, Europhys. Lett. 49, 143 (2000).

[7] M. P. Allen and D. J. Tildsley, Computer Simulations of Liquids (Clarendon Press, Oxford, 1987). 